\title{
Optimal design of spiral-wound membrane networks for gas separations
}

\author{
Runhong Qi, Michael A. Henson* \\ Department of Chemical Engineering, Louisiana State University, Baton Rouge, LA 70803-7303, USA \\ Received 13 January 1998; received in revised form 15 May 1998; accepted 22 May 1998
}

\begin{abstract}
An optimal design strategy for spiral-wound membrane networks based on an approximate permeator model and a mixedinteger nonlinear programming (MINLP) solution strategy is proposed. A general permeator system superstructure is used to embed a very large number of possible network configurations. The superstructure allows the development of a MINLP design strategy which simultaneously optimizes the permeator configuration and operating conditions to minimize an objective function which approximates the total annual process cost. Case studies for the separation of $\mathrm{CO}_{2} / \mathrm{CH}_{4}$ mixtures in natural gas treatment and enhanced oil recovery are presented. Permeator configurations are derived for different number of separation stages for both continuous and discrete membrane areas. The proposed approach provides an efficient methodology for the preliminary design of multi-stage membrane separation systems for binary gas mixtures. (C) 1998 Elsevier Science B.V. All rights reserved.
\end{abstract}

Keywords: Gas separations; Modules; Design; Optimization

\section{Introduction}

Membrane systems have become viable alternatives to conventional gas separation technologies such as pressure swing adsorption and cryogenic distillation. A particularly important application of membrane technology is the use of spiral-wound permeators to separate methane/carbon dioxide mixtures encountered in natural gas treatment and enhanced oil recovery. The economics of membrane separation processes depend critically on the process design. Single-stage systems have low capital costs, but they are appropriate only for moderate product purity and recovery

\footnotetext{
*Corresponding author. Tel.: +1-504-388-3690; fax: +1-504388-1476; e-mail: henson@nlc.che.lsu.edu
}

requirements. Multiple separation stages and recycle are required for more demanding applications. The design of a membrane system involves the determination of: (i) the configuration of the permeator network; and (ii) the operating conditions of the individual permeators.

Membrane systems currently are designed via a sequential procedure in which the permeator configuration is chosen by process heuristics and the operating conditions are determined using some type of optimization procedure. Many investigators have considered the design of multi-stage gas permeation systems [1-14]. Spillman et al. [4] design membrane systems to separate $\mathrm{CO}_{2} / \mathrm{CH}_{4}$ mixtures encountered in natural gas treatment and enhanced oil recovery. Several permeator configurations are optimized for 
a particular feed composition. Babcock et al. [5] evaluate the economics of single-stage and three-stage membrane systems for the natural gas treatment by providing comparisons with amine treatment processes. Bhide and Stern present detailed case studies of membrane separation systems for natural gas treatment [6,7] and oxygen enrichment of air [8,9]. A grid search method is used to optimize the operating conditions for several different configurations. Agrawal and $\mathrm{Xu}$ [11-14] develop a stepwise procedure for the synthesis of membrane cascades using limited numbers of recycle compressors. This approach is based on a master cascade which contains a large number of possible permeation stages and a specified number of recycle compressors. Substructure cascades are generated by eliminating unwanted recycle compressors and membrane stages using heuristic process analysis. A systematic method for optimizing the membrane cascades and their operating conditions is not presented.

Sequential design procedures are very inefficient because it is usually not feasible to enumerate and evaluate all possible network configurations. As a result, existing design techniques often yield suboptimal flowsheets. During the last decade, a wide variety of process design and synthesis problems have been solved by mathematical programming [15-17]. This approach utilizes rigorous optimization methods to systematically determine the process configuration and operating conditions. A popular approach is to postulate a superstructure which embeds many process configurations, each of which is a candidate for the optimal process flowsheet [18]. The superstructure is mathematically described by a model which contains both continuous and integer variables that represent operating conditions, as well as processing units and their interconnections. The mixed-integer nonlinear programming (MINLP) model is posed as a set of constraints in an optimization problem in which the total annual process cost usually is the objective function. An algorithm for solving the resulting MINLP problem has been developed and implemented as DICOPT $++[19]$ within the general algebraic modeling system (GAMS) [20]. MINLP techniques have allowed extensive progress in the synthesis of heat-exchanger networks, distillation column sequences, reactor networks, and mass-exchange networks [15-18].
For gas membrane separation systems, a major difficulty in applying MINLP synthesis techniques is that fundamental permeator models are comprised of differential-algebraic-integral equations with mixed boundary conditions. These models are too mathematically complex and computationally intensive to be utilized for MINLP design. An alternative approach is to develop approximate models which provide a more reasonable compromise between prediction accuracy and computational efficiency. Approximate models usually consist of a set of nonlinear algebraic equations which can be solved much more efficiently. Petterson and Lien [10] study the design of single-stage and multi-stage membrane systems using an algebraic model for hollow-fiber gas permeators. The permeator configurations are chosen a priori, and nonlinear programming (NLP) is used to optimize the operating conditions. However, the approximate permeator model does not yield accurate predictions when the feed concentration of the more permeable component is higher than 0.30.5 mol fraction. Recently, we proposed an approximate modeling technique for spiral-wound permeators separating binary [21] and multicomponent [22] gas mixtures. The model development is based on simplifying basic transport models which include permeate-side pressure drop. The resulting models are ideally suited for process design because the nonlinear algebraic equations can be solved very efficiently and yield excellent prediction accuracy over a wide range of operating conditions. We have used the approximate binary model to develop a NLP design strategy that allows systematic determination of the operating conditions for a specified permeator configuration [23].

In this paper, we utilize the same binary model to develop a process synthesis strategy for spiral-wound membrane systems which allows simultaneous optimization of the permeator network and operating conditions. The approach is based on a permeator system superstructure which efficiently embeds a very large number of possible network configurations. The superstructure is used to develop a MINLP design strategy which determines the membrane system that minimizes the approximate annual process cost. The methodology is applied to the separation of $\mathrm{CO}_{2} / \mathrm{CH}_{4}$ mixtures in natural gas treatment and enhanced oil recovery. Optimal separation systems are derived for 
different number of separation stages with both continuous and discrete membrane areas.

\section{Problem statement}

The problem of designing spiral-wound membrane systems for gas separations can be described as: given a feed mixture of known conditions, synthesize the minimum cost network of spiral-wound permeators and recycle compressors that separates the feed stream into products of specified compositions. The membrane properties and cost related parameters are assumed to be known.

The design task involves the determination of the optimal system configuration, as well as specification of the process unit sizes and operating conditions. As a first step, it is necessary to utilize a permeator model that is sufficiently accurate to predict the separation performance and computationally efficient for mathematical programming. The next step is to derive a permeator system superstructure which embeds all system configurations of practical interest, formulate the superstructure as a MINLP model, and develop a suitable solution strategy. To facilitate the subsequent development, the following assumptions are invoked:

1. The feed stream contains a binary gas mixture at a relatively high pressure.

2. The feed-side pressure for each stage is equal to the pressure of the feed stream.

3. The feed-side pressure drop is negligible for each stage.

4. There is no pressure drop between permeation stages.

5. The permeate stream pressures between stages are design variables, while the product permeate stream pressure is pre-determined.

6. All permeators and compressors operate at isothermal conditions.

\section{Spiral-wound permeator model}

The proposed optimal design strategy requires an accurate, yet computationally efficient, model of a spiral-wound gas permeator. In this section, we describe an approximate model which is derived directly from a fundamental cross-flow model [24] by assuming that the residual flow rate is constant in the direction of permeate flow. The approximate crossflow model is sufficiently accurate for spiral-wound permeators. A more detailed discussion of the model is given elsewhere [21].

The approximate model is comprised of four nonlinear algebraic equations. Eq. (1) describes the permeate-side pressure distribution,

$\gamma^{2}=\gamma_{0}^{2}+\frac{1}{2} C\left(1-\phi_{\mathrm{r}}\right)\left(1-h^{2}\right)$

where $\gamma$ is the ratio of the permeate-side and feed-side pressures, $\gamma_{0}$, is $\gamma$ at the permeate outlet, $\phi_{\mathrm{r}}$, the dimensionless residue gas flow rate and $h$, the dimensionless membrane leaf-length variable, and:

$C \equiv \frac{2 R_{\mathrm{g}} T \mu L U_{\mathrm{f}}}{W d_{m} B P^{2}}$

The remaining variables are defined in Section 7. The coefficient $C$ can be factored as follows:

$C=C^{\prime \prime} \frac{U_{\mathrm{f}}}{A P^{2}}$

where $U_{\mathrm{f}}$ is the feed gas flow rate, $P$, the feed-side pressure, $A$, the membrane area, and $C^{\prime \prime}$, a parameter that depends on the internal properties of the permeator. The second equation describes the effect of $\gamma$ and the local permeate-side concentration $y^{\prime}$ on the dimensionless feed-side flow rate $\phi$,

$\phi\left(\gamma, y^{\prime}\right)=\left(\frac{y^{\prime}}{y_{\mathrm{f}}^{\prime}}\right)^{a}\left(\frac{1-y^{\prime}}{1-y_{\mathrm{f}}^{\prime}}\right)^{b}\left(\frac{\alpha-(\alpha-1) y^{\prime}}{\alpha-(\alpha-1) y_{\mathrm{f}}^{\prime}}\right)$

where $y_{\mathrm{f}}^{\prime}$ is $y^{\prime}$ at the feed inlet, $\alpha$, the membrane selectivity, and:

$a=\frac{\gamma(\alpha-1)+1}{(\alpha-1)(1-\gamma)} \quad b=\frac{\gamma(\alpha-1)-\alpha}{(\alpha-1)(1-\gamma)}$

The dimensionless feed-side flow rate at the residue outlet can be written as,

$\phi_{\mathrm{r}}=\phi\left(\gamma, y_{\mathrm{r}}^{\prime}\right)$

where $y_{\mathrm{r}}^{\prime}$ is $y^{\prime}$ at the residue outlet.

The third equation describes the relation between the dimensionless permeation factor,

$R \equiv \frac{2 W L Q_{2} P}{d U_{\mathrm{f}}}=A \frac{Q_{2}}{d} \frac{P}{U_{\mathrm{f}}}$ 
and the local permeate concentration along the residue outlet $y_{\mathrm{r}}^{\prime}$ :

$$
\begin{aligned}
R= & \frac{1}{\alpha(1-\gamma)}\left\{\alpha-(\alpha-1) y_{\mathrm{f}}^{\prime}\right. \\
& \left.-\left[\alpha-(\alpha-1) y_{\mathrm{r}}^{\prime}\right] \phi\left(\gamma, y_{\mathrm{r}}^{\prime}\right)-(\alpha-1) I\left(\gamma, y_{\mathrm{r}}^{\prime}\right)\right\}
\end{aligned}
$$

Here $I\left(\gamma, y_{\mathrm{r}}^{\prime}\right)$ is an integral function which is approximated using Gaussian quadrature [25],

$I\left(\gamma, y_{\mathrm{r}}^{\prime}\right) \cong\left(y_{\mathrm{r}}^{\prime}-y_{\mathrm{f}}^{\prime}\right) \sum_{j=1}^{M} \phi\left(\gamma, y_{j}^{\prime}\right) w_{j}$

where $M$ is the number of quadrature points, $w_{\mathrm{j}}$, the quadrature weight at the quadrature point $\xi_{\mathrm{j}}$, and:

$y_{j}^{\prime}=y_{\mathrm{f}}^{\prime}+\xi_{j}\left(y_{\mathrm{r}}^{\prime}-y_{\mathrm{f}}^{\prime}\right)$

The fourth equation describes the relation between the local feed-side concentration $x$ and the local permeate-side concentration $y^{\prime}$ :

$\frac{y^{\prime}}{1-y^{\prime}}=\frac{\alpha\left(x-\gamma y^{\prime}\right)}{1-x-\gamma\left(1-y^{\prime}\right)}$

Simultaneous solution of Eqs. (1), (6), (8) and (11) with $x=x_{\mathrm{f}}$ at the quadrature point $h_{i}$ (see below) yields $\gamma\left(h_{i}\right), \phi_{r}\left(h_{i}\right), y_{\mathrm{r}}^{\prime}\left(h_{i}\right)$, and $y_{\mathrm{f}}^{\prime}\left(h_{i}\right)$. The local residue concentration $x_{\mathrm{r}}\left(h_{i}\right)$ is obtained from Eq. (11) with $y^{\prime}=y_{\mathrm{r}}^{\prime}\left(h_{i}\right)$.

The flow rate and concentration of the effluent permeate stream are calculated from the integral expressions that are approximated using the Gaussian quadrature. Under most conditions, a single quadrature point at $h_{1}=0.5$ is sufficient. In this case, the resulting equations are [21]:

$\theta_{0}=1-\phi_{\mathrm{r}}\left(h_{1}\right)$

$y_{0}=\frac{x_{f}-x_{\mathrm{r}}\left(h_{1}\right) \phi_{\mathrm{r}}\left(h_{1}\right)}{1-\phi_{\mathrm{r}}\left(h_{1}\right)}$

The flow rate and concentration of the effluent residue stream are determined from an overall material balance about the permeator. We have shown that the approximate model compares favorably with the fundamental model in terms of prediction accuracy [21]. The major advantage of the approximate model is that the nonlinear algebraic equations can be solved 200400 times faster than the fundamental differential- algebraic-integral equations using the shooting method [24].

\section{Optimal design strategy}

\subsection{Permeator superstructure}

The superstructure approach to process design provides a systematic framework for simultaneous optimization of process configuration and operating conditions [18]. Superstructures have been developed for a number of membrane separation systems, including reverse osmosis [26] and pervaporation [27] networks. For gas membrane separation systems, the basic components of the superstructure are permeators, compressors, stream mixers, and stream splitters. An ideal superstructure is sufficiently 'rich' to represent all process configurations of practical interest, yet sufficiently 'simple' to eliminate all unreasonable configurations.

The permeator system superstructure is derived as described below [18]. Note that each separation stage may be comprised of several permeators in parallel or in series.

1. The feed stream is split into individual feed streams for each permeation stage.

2. The inlet stream to a particular stage consists of: its individual feed stream; recycle streams obtained from the permeator's effluent streams; and recycle streams obtained from the effluent streams of all other stages.

3. For each stage, the permeate and residue streams are split into: recycle streams for the particular stage; recycle streams for all other stages; and streams that are sent to the final product mixers. The permeate recycle streams must be compressed to the feed pressure before being sent to the feed stream mixers.

4. The inlet streams for the final permeate (residue) mixer are obtained from the permeate (residue) streams of all stages.

As an illustration, the system superstructure for three separation stages is shown in Fig. 1. This superstructure is capable of representing a very large number of permeator configurations with three separation stages. Superstructures containing different number of separation stages are developed similarly. 


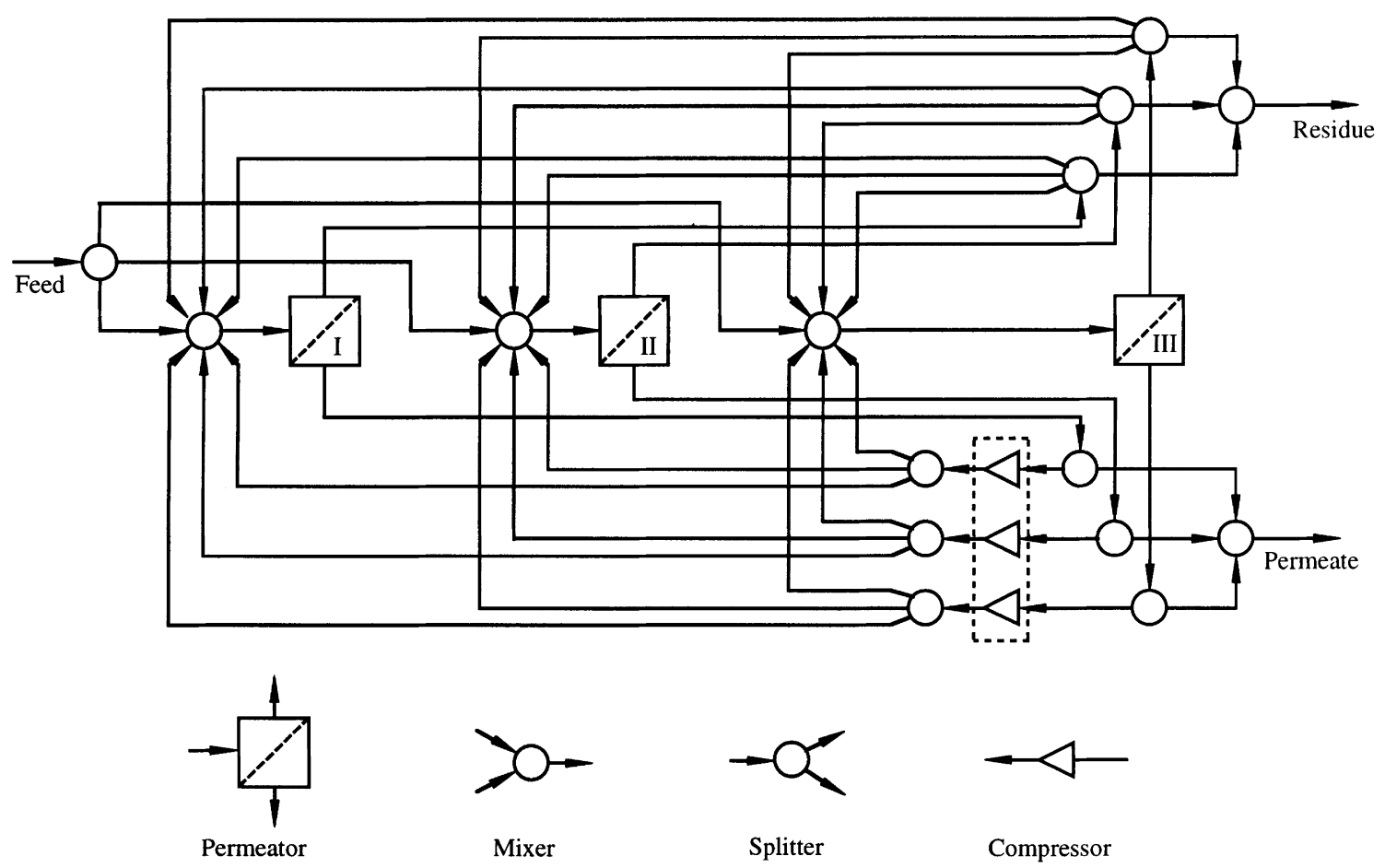

Fig. 1. Permeator system superstructure with three permeation stages.

\subsection{Mathematical formulation}

The permeator system superstructure is mathematically modeled using the general formulation:

$$
\begin{array}{lc}
\min : & c \boldsymbol{Z}+f(\boldsymbol{X}) \\
\text { s.t. }: & U_{1} \boldsymbol{Z}+h(\boldsymbol{X})=0 \\
& U_{2} \boldsymbol{Z}+g(\boldsymbol{X}) \leq 0 \\
& \boldsymbol{X} \in R^{n} \\
& \boldsymbol{Z} \in\{0,1\}^{l}
\end{array}
$$

where: $\boldsymbol{X}$ is a vector of $n$ continuous variables that represent flow rates, pressures, and compositions of the process streams, as well as continuous properties of the process units, $\boldsymbol{Z}$, a vector of $l$ binary variables that denote the existence $\left(\boldsymbol{Z}_{i}=1\right)$ or non-existence $\left(\boldsymbol{Z}_{i}=0\right)$ of the process units and connections, as well as the discrete properties of the process units, $c \mathbf{Z}+f(\boldsymbol{X})$, an objective function which approximates the annual cost of the membrane system, $U_{1} \boldsymbol{Z}+h(\boldsymbol{X})$, $m$ equality constraints that denote material balances, permeator model equations, and parameter definitions and $U_{2} \boldsymbol{Z}+g(\boldsymbol{X}), p$ inequality constraints which corre- spond to the separation requirements, operational restrictions, and logical constraints. Note that all binary variables appear linearly, while the continuous variables may appear nonlinearly in the functions $f(\boldsymbol{X})$, $g(\boldsymbol{X})$, and $h(\boldsymbol{X})$.

\subsubsection{Annual process cost}

The optimal design of a membrane system entails a tradeoff between capital investments and operating expenses. The annual process cost should take into account capital investments associated with permeators and compressors, as well as operating expenses due to the replacement of membrane elements, maintenance, consumption of utilities, and product losses. Depending on the application, the calculation of process costs requires different levels of accuracy. Here we focus on $\mathrm{CO}_{2} / \mathrm{CH}_{4}$ separations and utilize the approximate costing procedures presented by Spillman et al. [4] and Babcock et al. [5]

The fixed capital investment is the installed equipment cost of membrane vessels and compressors. Note that membrane housing is a capital cost, but the 
replacement of membrane elements is treated as an operating expense. Both membrane housing and the replacement of the elements are determined by the membrane area. Auxiliary costs associated with pipes, fittings, and assembly are included in the membrane housing cost. As a result, the fixed capital investment $\left(F_{\mathrm{fc}}\right)$ is only a function of membrane area $(A)$ and compressor power $\left(W_{\mathrm{cp}}\right)$ for each stage $(n)$ :

$F_{\mathrm{fc}}=\sum_{n=1}^{N}\left(f_{\mathrm{mh}} A_{n}+f_{\mathrm{cp}} W_{\mathrm{cp}, n} / \eta_{\mathrm{cp}}\right)$

where $N$ is the number of separation stages. The remaining parameters and variables are defined in Section 7. The compressor power for each stage is calculated by assuming ideal gas behavior and isothermal compression [28]:

$W_{\mathrm{cp}, n}=R_{\mathrm{g}} T\left(\sum_{m=1}^{N} V_{\mathrm{b}, m, n}\right) \ln \left(\frac{P}{p_{0, n}}\right), n=1, \ldots, N$

where $V_{\mathrm{b}, m, n}$ is the permeate recycle stream from the $n$ th stage to $m$-th stage. The working capital is taken as a fixed percentage $\left(f_{\mathrm{wk}}\right)$ of the fixed capital, and the annual capital charge $\left(F_{\mathrm{cc}}\right)$ is calculated by annualizing the fixed and working capitals:

$F_{\mathrm{cc}}=f_{\mathrm{cc}}\left(1+f_{\mathrm{wk}}\right) F_{\mathrm{fc}}$

The annual operating costs include membrane replacement expense $\left(F_{\mathrm{mr}}\right)$ :

$F_{\mathrm{mr}}=\frac{f_{\mathrm{mr}}}{t_{\mathrm{m}}} \sum_{n=1}^{N} A_{n}$

maintenance expense $\left(F_{\mathrm{mt}}\right)$ :

$F_{\mathrm{mt}}=f_{\mathrm{mt}} F_{\mathrm{fc}}$

cost of utilities $\left(F_{\mathrm{ut}}\right)$ :

$F_{\mathrm{ut}}=\frac{f_{\mathrm{sg}} t_{\mathrm{wk}}}{f_{\mathrm{hv}} \eta_{\mathrm{cp}}} \sum_{n=1}^{N} W_{\mathrm{cp}, n}$

and value of product losses $\left(F_{\mathrm{pl}}\right)$ :

$F_{\mathrm{pl}}=f_{\mathrm{sg}} t_{\mathrm{wk}} V_{\mathrm{pt}} \frac{1-y_{\mathrm{pt}}}{1-x_{\mathrm{pt}}}$

The annual process cost $(F)$ is taken as the sum of the capital charge and operating expenses divided by process capacity, which is expressed as:

$F=\frac{F_{\mathrm{cc}}+F_{\mathrm{mr}}+F_{\mathrm{mt}}+F_{\mathrm{ut}}+F_{\mathrm{pl}}}{U_{f 00} t_{\mathrm{wk}}}$

The annual process cost $(F)$ is used as the objective function, which is minimized subject to various types of constraints described below. It is important to note that the above calculation procedure only provides an estimate of the annual process cost. As a result of the approximate permeator model and the approximate economic analysis, the MINLP strategy yields preliminary designs. These designs can be used as a basis for more detailed analysis.

\subsubsection{Material balance constraints}

Material balance constraints are imposed on: (i) splitters for the initial feed stream and the outlet streams of each stage; (ii) mixers for the inlet streams of each stage and the inlet streams for the final products; and (iii) each permeation stage. For an $N$ stage system, material balances on the splitters can be expressed as:

$$
\begin{aligned}
& U_{\mathrm{f} 00}=\sum_{n=1}^{N} U_{\mathrm{f} 0, n} \\
& U_{0, n}=U_{\mathrm{p}, n}+\sum_{m=1}^{N} U_{\mathrm{b}, m, n}, n=1, \ldots, N \\
& V_{0, n}=V_{\mathrm{p}, n}+\sum_{m=1}^{N} V_{\mathrm{b}, m, n}, n=1, \ldots, N
\end{aligned}
$$

where, $U_{\mathrm{fo0}}$ is the total fresh feed flow rate, $U_{\mathrm{f} 0, n}$, the fresh feed flow rate for stage $n, U_{0, n}\left(V_{0, n}\right)$, the total outlet residue (permeate) flow rate for stage $n$, $U_{\mathrm{p}, n}\left(V_{\mathrm{p}, n}\right)$, the residue (permeate) flow rate of the final product from stage $n$, and $U_{\mathrm{b}, m, n}\left(V_{\mathrm{b}, m, n}\right)$, the residue (permeate) flow rate of the recycle stream from the $n$ th stage to $m$-th stage. Note that only overall material balances are needed because splitters do not change stream compositions.

For the stream mixers, both overall material balances and component balances are necessary. Material balances for the inlet mixer of stage $n$ are written as:

$U_{\mathrm{f}, n}=U_{\mathrm{f} 0, n}+\sum_{m=1}^{N}\left(U_{\mathrm{b}, n, m}+V_{\mathrm{b}, n, m}\right), \quad n=1, \ldots, N$ 


$$
\begin{gathered}
U_{\mathrm{f}, n} x_{\mathrm{f}, n}=U_{\mathrm{f} 0, n} x_{\mathrm{f} 0}+\sum_{m=1}^{N}\left(U_{\mathrm{b}, n, m} x_{0, m}+V_{\mathrm{b}, n, m} y_{0, m}\right), \\
n=1, \ldots, N
\end{gathered}
$$

Note that the recycle streams are taken from the $m$-th stage and terminate at the $n$-th stage. Material balances for the product mixers are expressed as:

$U_{\mathrm{pt}}=\sum_{n=1}^{N} U_{\mathrm{p}, n}$

$U_{\mathrm{pt}} x_{\mathrm{pt}}=\sum_{n=1}^{N} U_{\mathrm{p}, n} x_{0, n}$

$V_{\mathrm{pt}}=\sum_{n=1}^{N} V_{p, n}$

$V_{\mathrm{pt}} y_{\mathrm{pt}}=\sum_{n=1}^{N} V_{p, n} y_{0, n}$

where, $U_{\mathrm{pt}}$ and $x_{\mathrm{pt}}$ are the total flow rate and concentration of the final residue product, and $V_{\mathrm{pt}}$ and $y_{\mathrm{pt}}$ are the total flow rate and concentration of the final permeate product. Material balances about each permeation stage yield:

$U_{\mathrm{f}, n}=U_{0, n}+V_{0, n}, n=1, \ldots, N$

$U_{\mathrm{f}, n} x_{f, n}=U_{0, n} x_{0, n}+V_{0, n} y_{0, n}, \quad n=1, \ldots, N$

\subsubsection{Permeator model constraints}

The permeator model constraints are the approximate permeator model equations written for each stage. Some of the equations are manipulated to facilitate computer implementation. The resulting model equations are presented in the Appendix A.

\subsubsection{Operating requirement constraints}

Constraints are needed to ensure that the product streams satisfy the separation requirements. In $\mathrm{CO}_{2} /$ $\mathrm{CH}_{4}$ separations, minimum purity requirements are placed on the final residue and permeate streams. In addition, a constraint which expresses that the permeate pressure for each stage must be at least as high as the pressure of the final permeate stream is required. These constraints are expressed as:

$x_{\mathrm{pt}} \leq x_{\mathrm{out}}$ $y_{\mathrm{pt}} \geq y_{\text {out }}$

$p_{0, n} \geq p_{\text {out }}$

Depending on the application, some of the constraints may be relaxed. For example, only Eqs. (34) and (36) are required for natural gas treatment since the $\mathrm{CO}_{2}$ enriched permeate stream has no value.

\subsubsection{Discrete membrane area constraints}

A typical spiral-wound permeator is comprised of several membrane elements placed in a cylindrical steel shell. A permeator shell normally is capable of holding from one to six spiral-wound membrane elements. Membrane area is adjusted by changing the number of elements or by connecting several permeators in series or parallel. As a result, membrane area can be considered as a discrete variable:

$A_{n}=A_{0} N_{n}^{A}, n=1, \ldots, N$

where, $A_{0}$ is the element membrane area and $N^{A}\left(N^{A L} \leq N^{A} \leq N^{A U}\right)$ is the number of elements. Since integer variables cannot be handled directly by existing MINLP algorithms, the element number $N^{A}$ must be expressed in terms of binary variables. One way to convert the integer variables $N^{A}$ to binary variables $Z^{A}$ is to use the following expression [18]:

$N_{n}^{A}=N_{n}^{A L}+\sum_{k=1}^{K} 2^{k-1} \boldsymbol{Z}_{n, k}^{A}, n=1, \ldots, N$

where $K$ is the minimum number of binary variable needed:

$K=1+\operatorname{int}\left\{\frac{\log \left(N^{A U}-N^{A L}\right)}{\log (2)}\right\}$

Note that Eq. (39) is used only to calculate the value of $K$; it is not used as a constraint equation. In the following case studies, we choose $N^{A L}=1$ and $N^{A U}=15$ to 30 , which yield $K=4$ or 5 . When the membrane area is regarded as a continuous variable, the constraints Eqs. (37) and (38) are not utilized.

\subsubsection{Other constraints}

Several other types of constraints are required to have a well defined optimization problem. These include logic constraints on the binary variables, as well as non-negativity and integrality constraints on both the continuous and binary variables. A 
description of these constraints is included in the Appendix A.

\subsection{Solution strategy}

The MINLP design model is solved using the algorithm of Viswanathan and Grossmann [19], which is available in GAMS [20] as the solver DICOPT ++ . The solution technique is based on an outer approximation approach in which the MINLP problem is decomposed into a series of NLP and MILP subproblems [29]. These subproblems can be solved using any NLP and MILP solvers that run in the GAMS environment. In this paper, CONOPT2 is used for the NLP problem and XA is used for the MILP problem. It is important to note that the MINLP formulation usually yields a nonconvex optimization problem. As a result, the solution obtained represents a local optimum. We address this problem by initializing the variables at several different points, setting reasonable bounds on variables, and adjusting the DICOPT ++ options to facilitate convergence to the global optimum.

\section{Case studies}

The MINLP design strategy is used to derive optimal permeator networks for natural gas treatment and enhanced oil recovery applications. The nominal economic parameters and operating conditions are obtained from Spillman et al. [4], Babcock et al. [5], and Lee et al. [30,31].

- Operating conditions

- natural gas processing capacity:

$U_{\mathrm{fO} 0}=10 \mathrm{~mol} / \mathrm{s}\left(19353 \mathrm{~m}^{3} /\right.$ day $)$,

- feed pressure: $P=3.5 \mathrm{MPa}$,

- feed $\mathrm{CO}_{2}$ concentration: $x_{\mathrm{f} 0}=0.20$,

- product permeate pressure: $p_{\text {out }}=$

$0.105 \mathrm{MPa}$,

- temperature: $T=40^{\circ} \mathrm{C}$,

- working time: $t_{\mathrm{wk}}=300$ days/year.

- Operating requirements

than $2 \%$

- product residue $\mathrm{CO}_{2}$ concentration less

- product permeate $\mathrm{CO}_{2}$ concentration greater than $95 \%$ (enhanced oil recovery only)

- outlet permeate pressure for each stage not less than $0.105 \mathrm{MPa}$.
- Membrane properties

$\mathrm{MPa} \mathrm{m}{ }^{2} \mathrm{~s}$,

- $\mathrm{CH}_{4}$ permeability: $Q_{2} / d=1.48 \times 10^{-3} \mathrm{~mol} /$

- $\mathrm{CO}_{2} / \mathrm{CH}_{4}$ selectivity: $\alpha=20$,

- pressure parameter: $\mathrm{C}^{\prime \prime}=9.32 \mathrm{MPa}^{2} \mathrm{~m}^{2} \mathrm{~s} /$ mol.

- Capital investment

— membrane housing: $f_{\mathrm{mh}}=200 \$ / \mathrm{m}^{2}$ membrane,

- gas-powered compressors: $f_{\mathrm{cp}}=1000 \$ / \mathrm{KW}$,

- compressor efficiency: $\eta_{\mathrm{cp}}=70 \%$,

- working capital: $f_{\mathrm{wk}}=10 \%$ of fixed capital investment,

- capital charge: $f_{\mathrm{cc}}=27 \%$ per year.

- Operating expenses

membrane,

- membrane replacement: $f_{\mathrm{mr}}=90 \$ / \mathrm{m}^{2}$

- membrane lifetime: $t_{\mathrm{m}}=3$ years,

- maintenance: $f_{\mathrm{mt}}=5 \%$ of fixed capital

investment per year,

- utility and sale gas price: $f_{\mathrm{sg}}=35 \$ / \mathrm{Km}^{3}$, $\mathrm{m}^{3}$,

- sales gas gross heating value: $f_{\mathrm{hv}}=43 \mathrm{MJ} /$

- lost $\mathrm{CH}_{4}$ is converted to sales gas value.

The gas volumes are calculated at standard conditions of $0.102 \mathrm{MPa}$ and $273 \mathrm{~K}$. The pressure parameter $C^{\prime \prime}$ is an estimated value based on experimental data $[21,30]$. In this work, we allow the membrane area to be both a continuous variable and a discrete variable.

\subsection{Natural gas treatment}

For natural gas treatment, the $\mathrm{CO}_{2}$ concentration of the residue product must be less than $2 \%$. No constraint is placed on the permeate concentration because the permeate stream is a low grade fuel or a waste gas. Flowsheets with two, three and four separation stages are synthesized to produce process configurations and operating conditions which minimize the annual cost. A configuration with continuous membrane area provides a lower bound on the annual cost for a particular number of separation stages. A configuration with discrete membrane area generally will yield a higher process cost, but the resulting flowsheet is more realistic.

The optimal configuration and operating conditions for the two-stage system with continuous membrane area are shown in Fig. 2, while the corresponding 


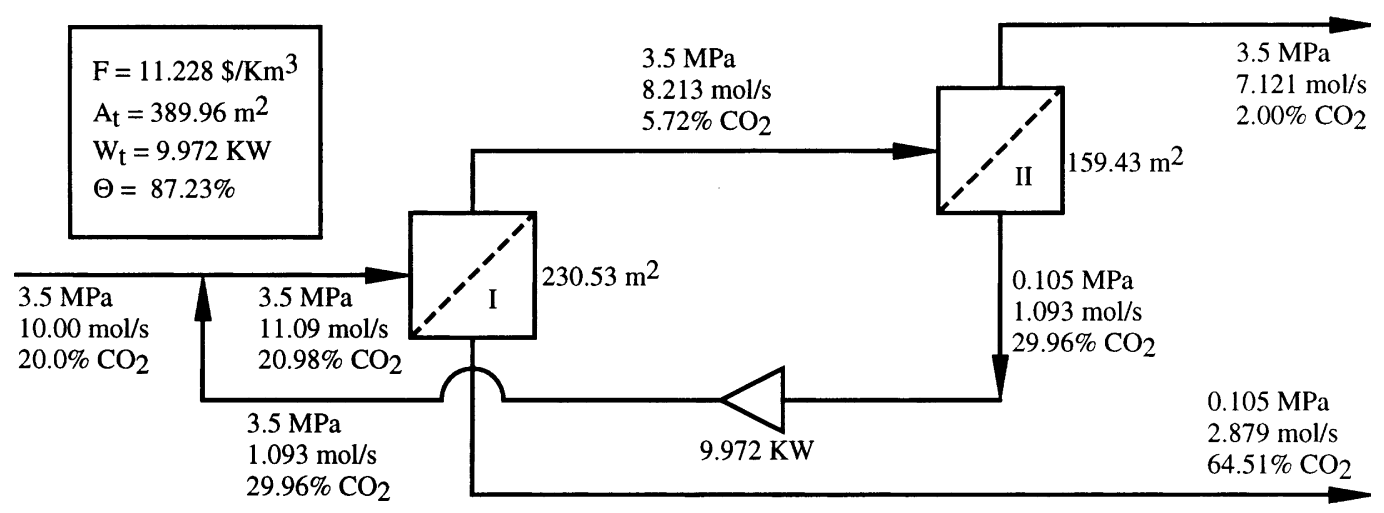

Fig. 2. Two-stage system with continuous membrane area (natural gas treatment).

results for a discrete membrane element area of $20 \mathrm{~m}^{2}$ are shown in Fig. 3. Note that the system configurations for the two cases are identical, while the operating conditions are significantly different. As expected, the process cost for the discrete area case is slightly higher than that for the continuous area case. The membrane area for each individual stage is quite different, but the total membrane area is very similar. The compressor power and $\mathrm{CH}_{4}$ recovery also differ because of the different distribution of membrane area. Note that both configurations satisfy the $98 \%$ $\mathrm{CH}_{4}$ purity constraint placed on the product residue stream.

Fig. 4 shows the optimal configuration and operating conditions for the three-stage system with continuous membrane area. This design represents a slight modification of the two-stage configuration (Fig. 2) in which a small third-stage permeator is used to separate the second-stage permeate stream. The total process cost is slightly lower than that of the two-stage system because of increased $\mathrm{CH}_{4}$ recovery. The configuration and operating conditions for the three-stage system with discrete membrane element area of $10 \mathrm{~m}^{2}$ are shown in Fig. 5. In this case, the system configuration is different from that obtained for the continuous area case (Fig. 4). The discrete area configuration contains a relatively large third stage which separates the second-stage permeate stream; this makes recycling of the third-stage residue stream unnecessary. The process cost is slightly higher than that of the continuous area case. Fig. 6 shows the design that results for a membrane element area of $20 \mathrm{~m}^{2}$. This configuration is different from that obtained for continuous membrane area (Fig. 4) and a discrete area of $10 \mathrm{~m}^{2}$ (Fig. 5). The design in Fig. 6 is a slight modification of the continuous area design obtained by collecting a

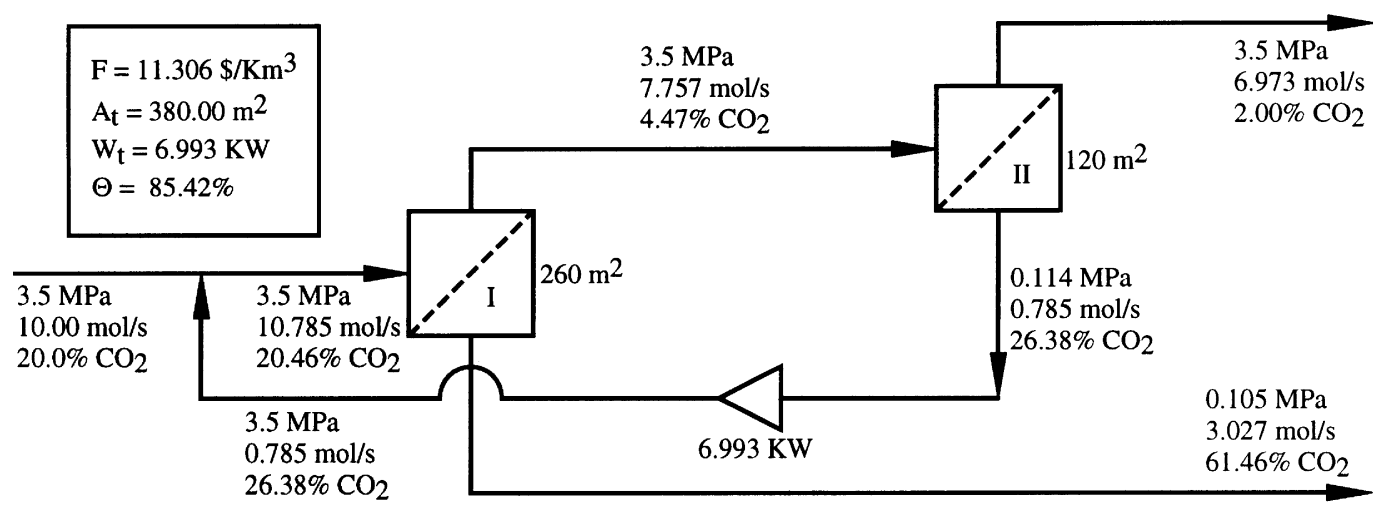

Fig. 3. Two-stage system with membrane element area of $20 \mathrm{~m}^{2}$ (natural gas treatment). 


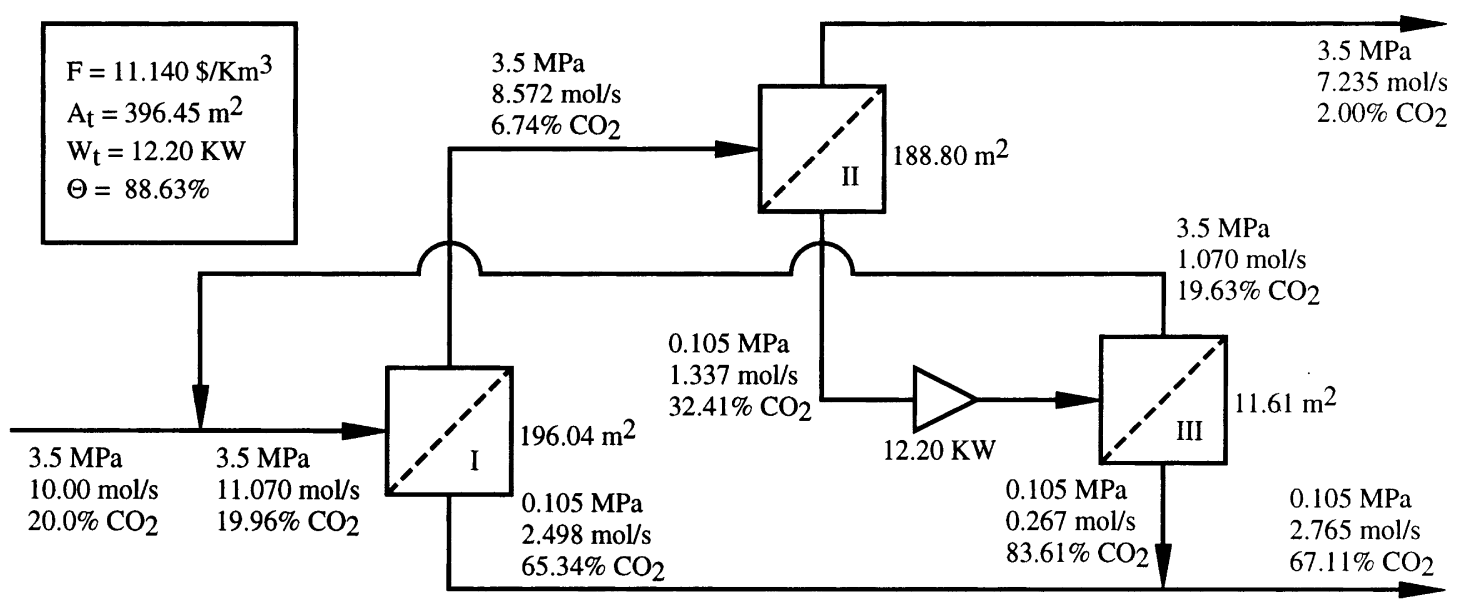

Fig. 4. Three-stage system with continuous membrane area (natural gas treatment).

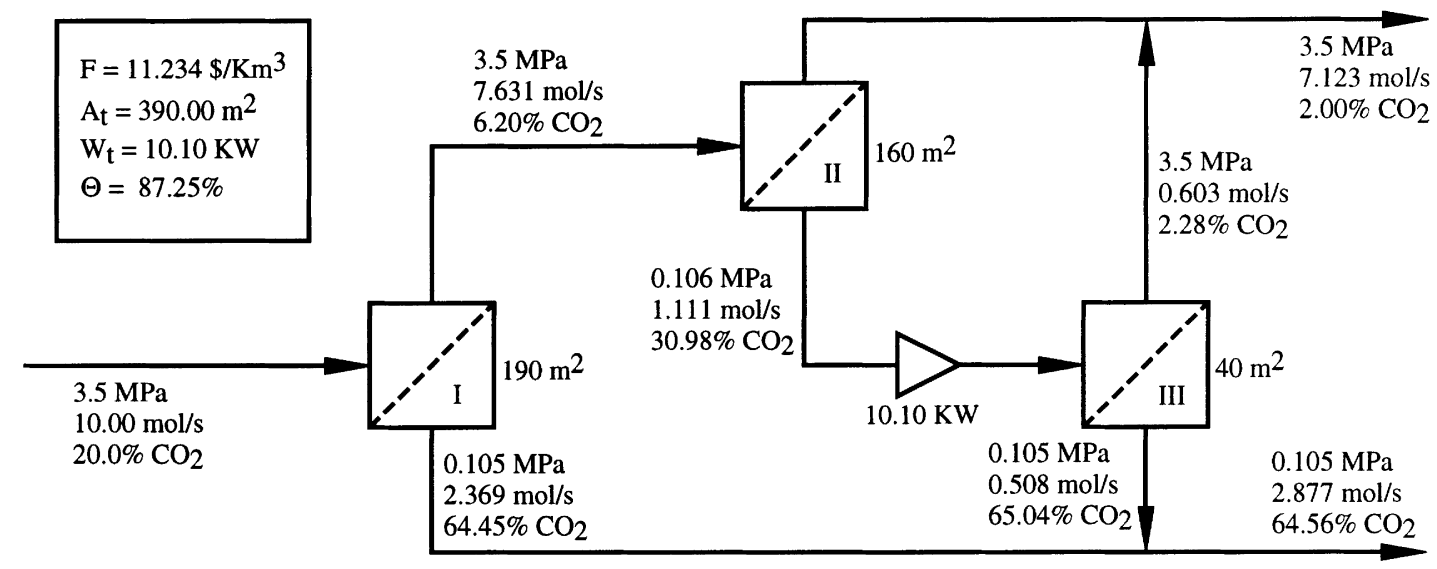

Fig. 5. Three-stage system with membrane element area of $10 \mathrm{~m}^{2}$ (natural gas treatment).

small portion of the second-stage permeate stream as the final product, while the remainder of the permeate stream is separated in the third stage. Although the process costs for the two configurations are very comparable, the configuration in Fig. 6 may not be very practical because the flow rate of the product stream $(0.027 \mathrm{~mol} / \mathrm{s})$ is very small compared to the flow rate of the second-stage permeate stream $(1.436 \mathrm{~mol} / \mathrm{s})$. Additional logic constraints are required to avoid this type of situation. It is important to note that the configuration obtained for an element area of $10 \mathrm{~m}^{2}$ (Fig. 5) has a higher process cost than that obtained with an element area of $20 \mathrm{~m}^{2}$ (Fig. 6). This occurs because more binary variables are required to represent the constraint Eq. (38) when small element areas are used. Apparently, different local optima were found for the two cases.

The optimal configuration and operating conditions for the four-stage system with continuous membrane area are presented in Fig. 7. This configuration is similar to that obtained for the three-stage system (Fig. 4), except that the third stage of Fig. 4 is divided into two smaller stages in Fig. 7. For discrete membrane element areas of $10 \mathrm{~m}^{2}$ and $20 \mathrm{~m}^{2}$, optimal solutions for the four-stage system could not be obtained because membrane area and flow rate differences are too large to allow convergence of the MINLP. 


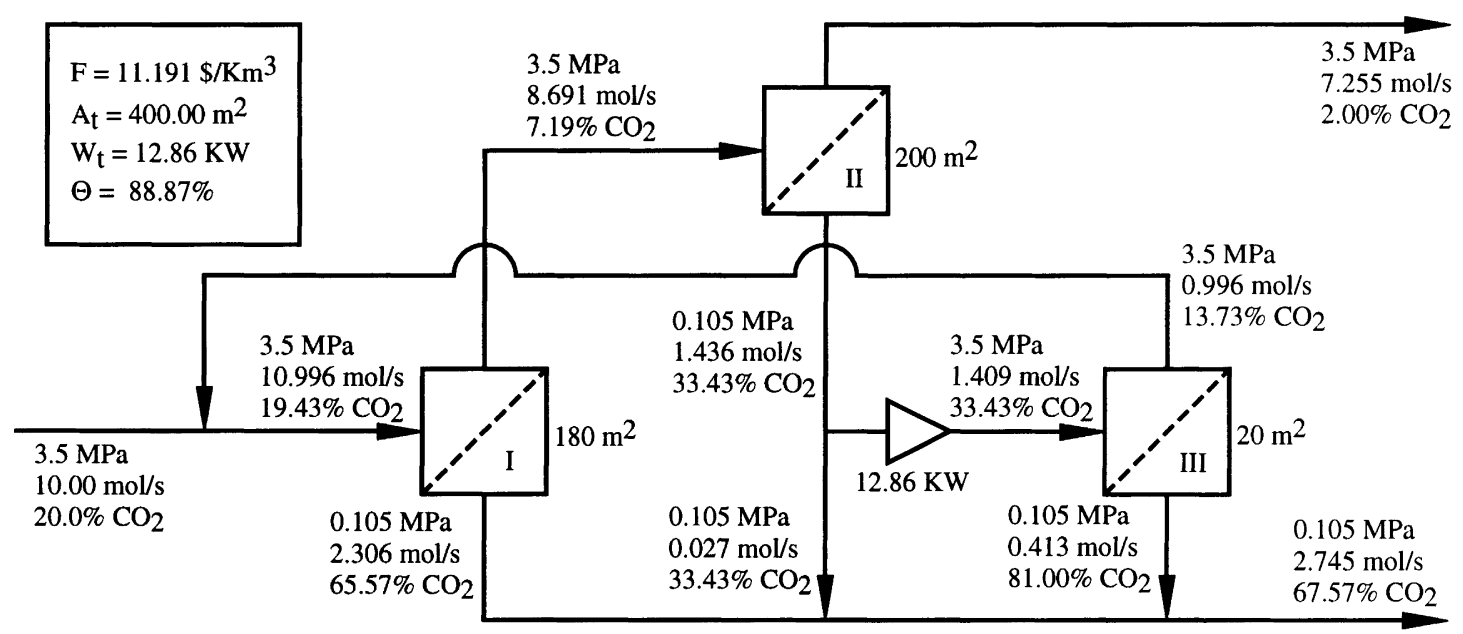

Fig. 6. Three-stage system with membrane element area of $20 \mathrm{~m}^{2}$ (natural gas treatment).

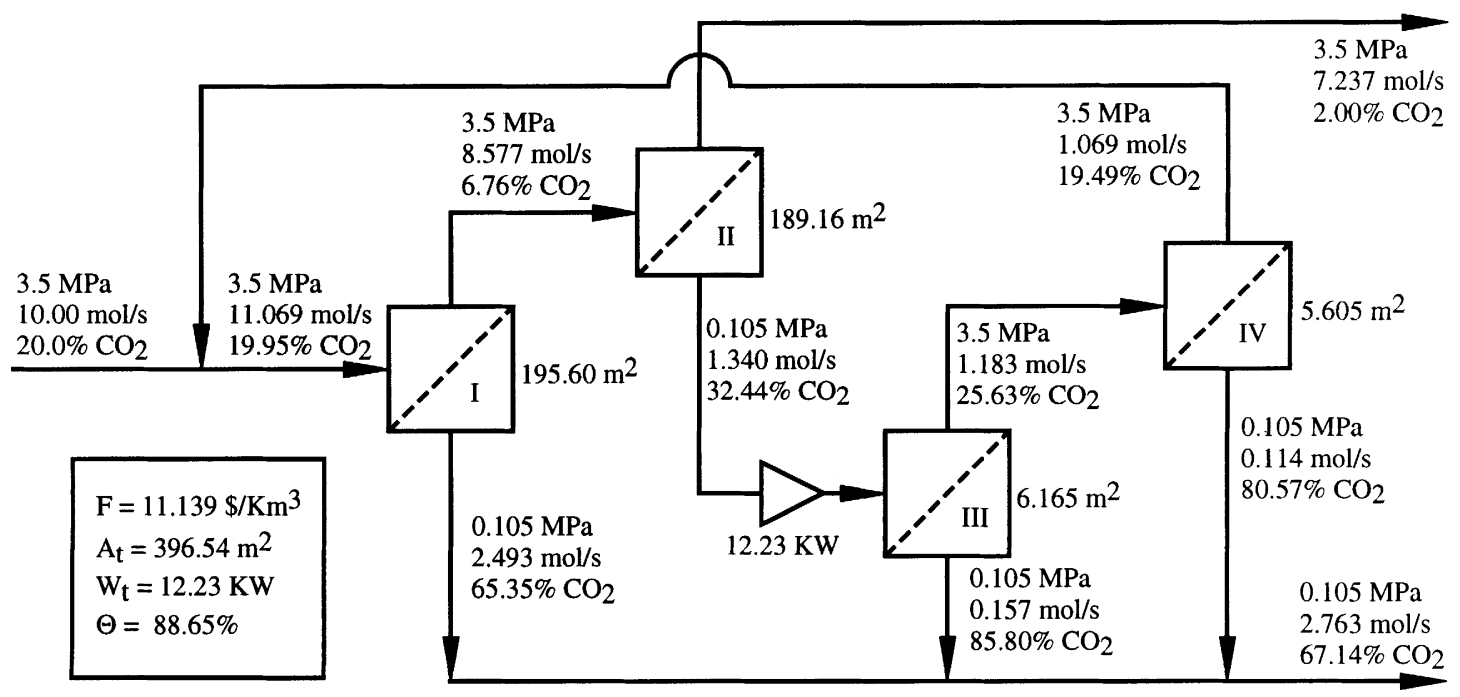

Fig. 7. Four-stage system with continuous membrane area (natural gas treatment).

Comparing the configurations and operating conditions shown in Figs. 2-7, we note that the process costs and total membrane areas of the designs are very similar even though membrane areas of individual stages and operating conditions are quite different. In particular, increasing the number of membrane stages does not affect the process cost significantly. The single purity constraint on the residue concentration results in a large number of degrees of freedom, which allows the total membrane area to be allocated differently with similar overall process costs.

\subsection{Enhanced oil recovery}

In $\mathrm{CO}_{2} / \mathrm{CH}_{4}$ separations for enhanced oil recovery, both the residue and permeate streams must satisfy composition requirements. As in the natural gas treatment case, we design optimal separation networks for two-stage, three-stage, and four-stage membrane systems. The same nominal parameters and feed conditions are used in this application.

Fig. 8 shows the optimal design for the two-stage system with continuous membrane area, while Fig. 9 


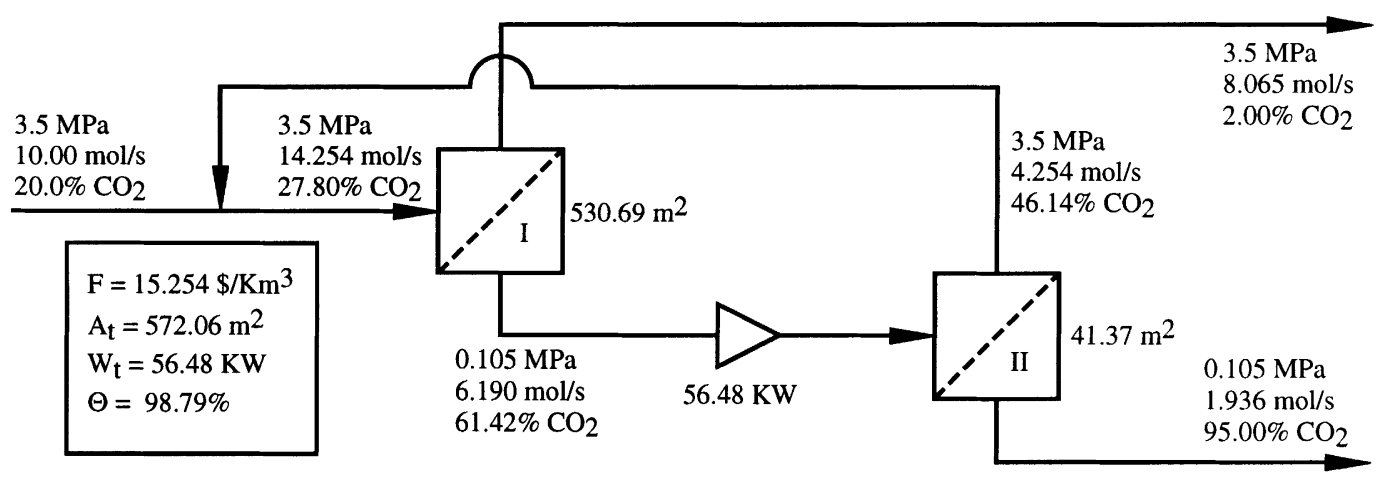

Fig. 8. Two-stage system with continuous membrane area (enhanced oil recovery).

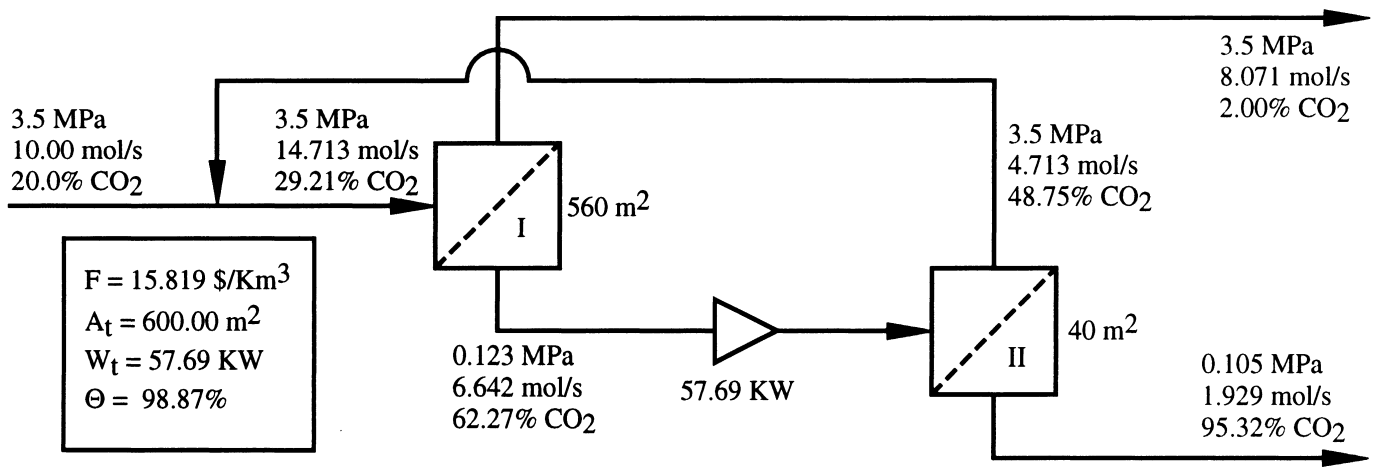

Fig. 9. Two-stage system with membrane element area of $20 \mathrm{~m}^{2}$ (enhanced oil recovery).

shows the optimal design for a discrete membrane element area of $20 \mathrm{~m}^{2}$. The configurations are identical, but the total membrane area is slightly larger for the discrete area case. It is interesting to note that the larger membrane area in the discrete case results in over-separation of the final permeate product. Optimal designs for the three-stage system with continuous membrane area and discrete membrane element area of $20 \mathrm{~m}^{2}$ are shown in Figs. 10 and 11, respectively. The two configurations are identical with recycle of both permeate and residue streams. A detailed investigation of this configuration with continuous membrane area has been presented in a previous paper [23]. Using discrete membrane area significantly changes the distribution of the membrane area and the operating conditions, but it has little effect on the total membrane area. Note that the permeate purity constraint is exceeded in the discrete area case. Figs. 12 and 13 show optimal designs for the four-stage system with continuous membrane area and discrete mem- brane element area of $20 \mathrm{~m}^{2}$, respectively. The configurations are identical and differ from the three-stage configurations (Figs. 10 and 11) in that the fourthstage is used to further separate the second-stage permeate stream and the third-stage residue stream before recycling. As before, the discrete area design exceeds the permeate purity constraint.

The results in Figs. 8-13 demonstrate that increasing the number of membrane stages can significantly decrease the process cost. However, the separation requirements on both the residue and permeate streams severely restrict the optimal process configuration and operating conditions.

\section{Conclusions}

An optimal design strategy for spiral-wound gas separation systems based on an algebraic permeator model and mixed-integer nonlinear programming 


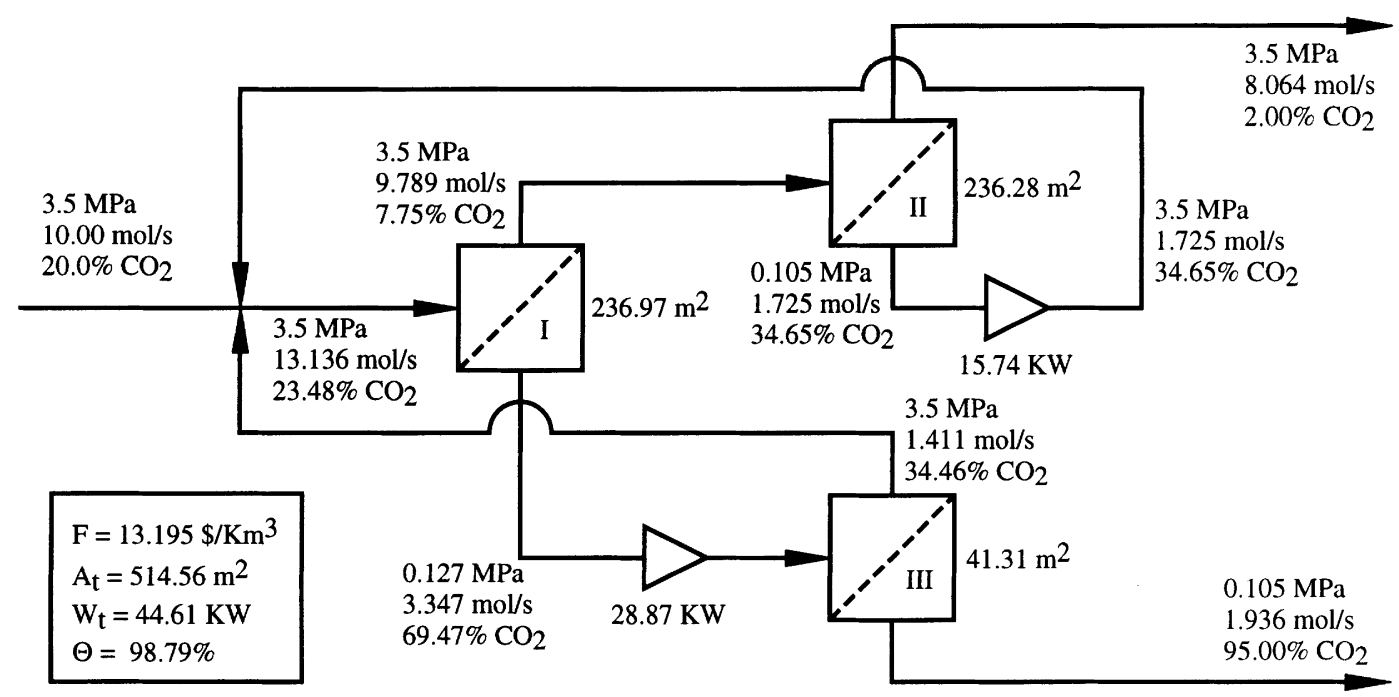

Fig. 10. Three-stage system with continuous membrane area (enhanced oil recovery).

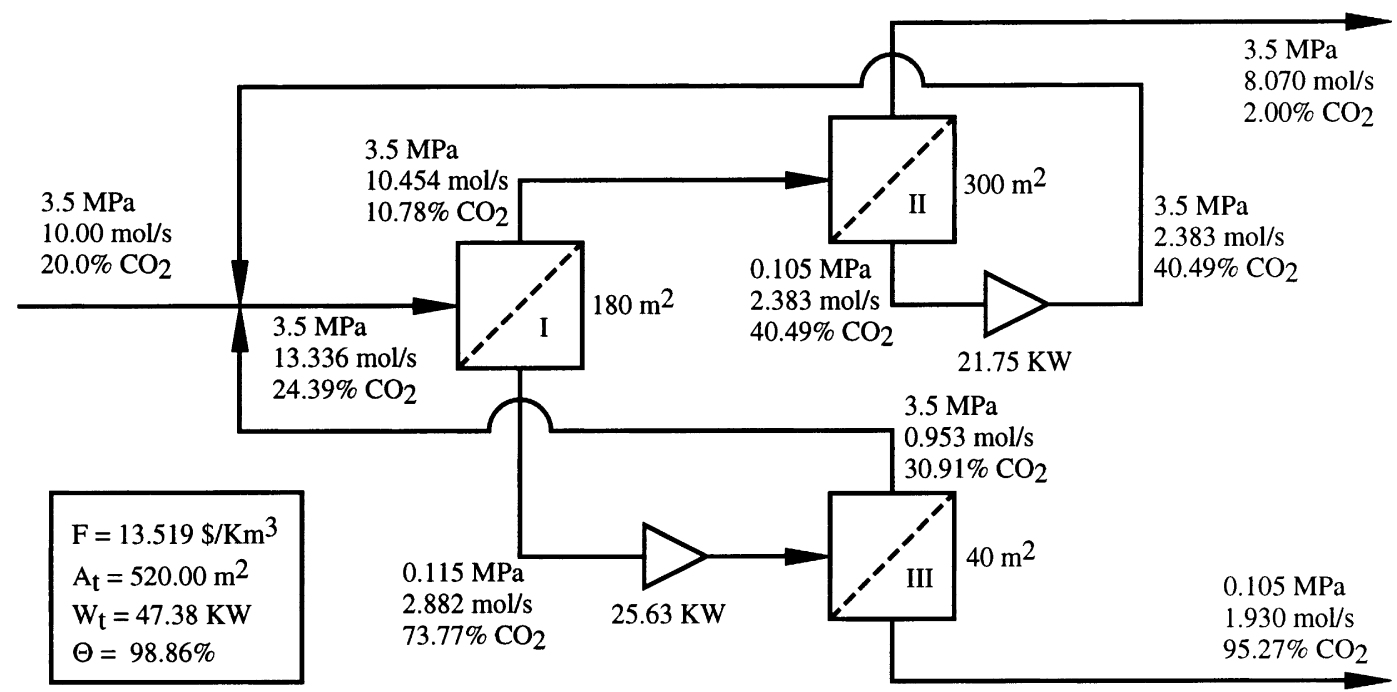

Fig. 11. Three-stage system with membrane element area of $20 \mathrm{~m}^{2}$ (enhanced oil recovery).

(MINLP) has been developed. The proposed process synthesis approach utilizes a permeator system superstructure which embeds a very large number of possible network configurations. The superstructure is formulated as a MINLP problem and solved using standard optimization tools to yield the system configuration and operating conditions which minimize the annual process cost. Case studies for the separation of $\mathrm{CO}_{2} / \mathrm{CH}_{4}$ mixtures in natural gas treatment and enhanced oil recovery have been presented. Optimal designs based a reasonable estimation of process costs are derived for different number of membrane stages with both continuous and discrete membrane area. The results demonstrate that the proposed design methodology provides an effective tool for preliminary design of multi-stage membrane separation systems. 


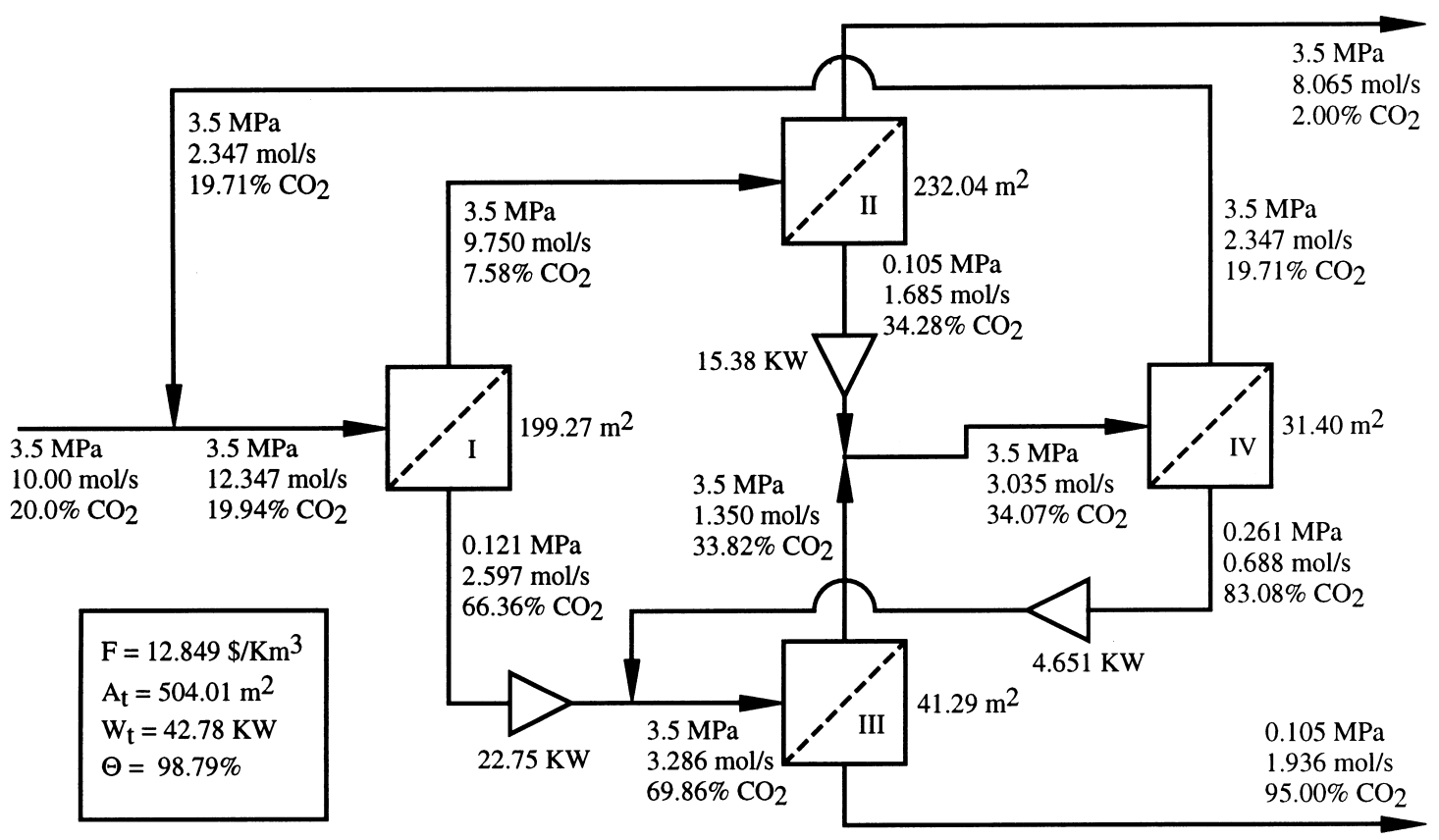

Fig. 12. Four-stage system with continuous membrane area (enhanced oil recovery).

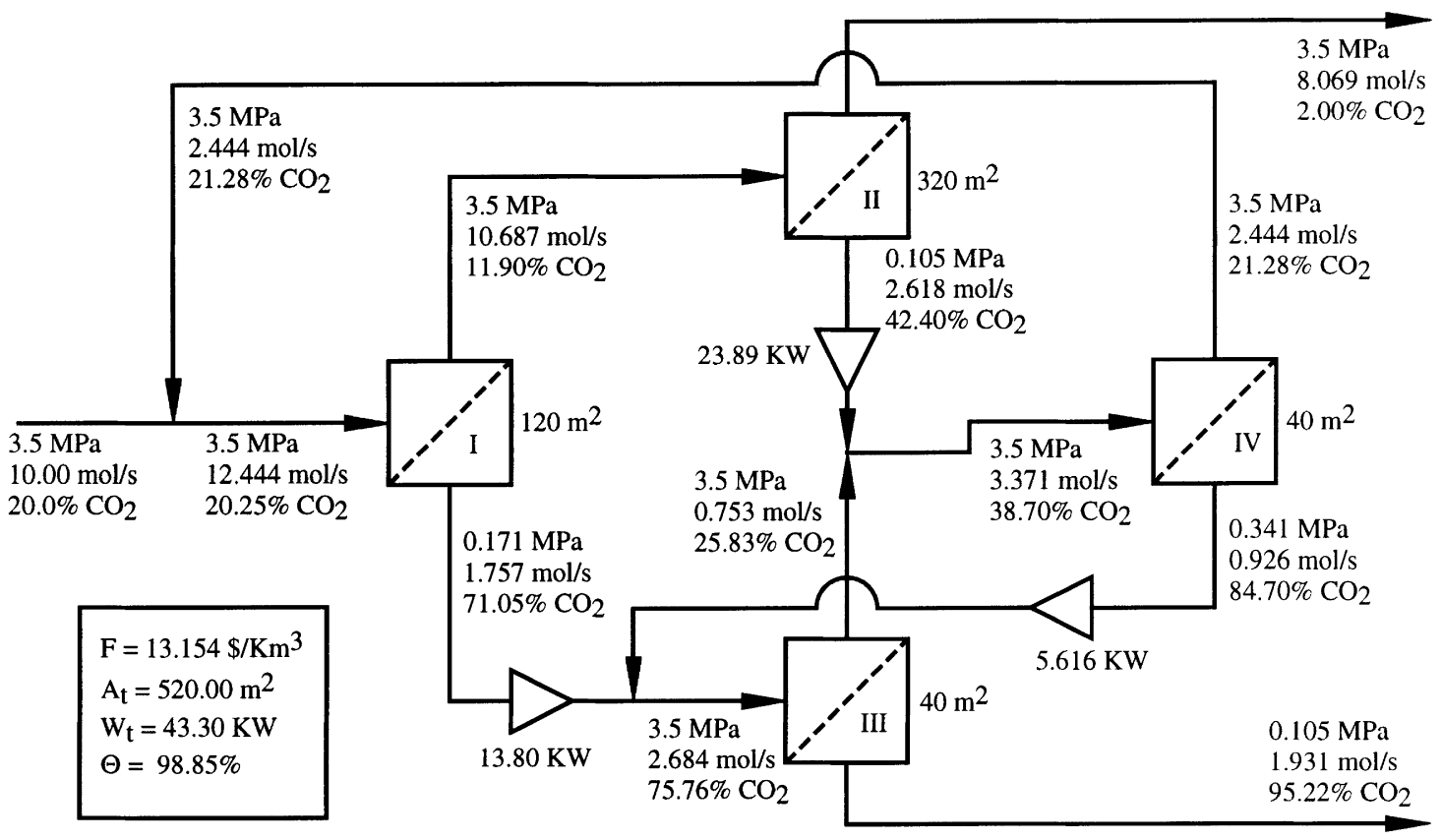

Fig. 13. Four-stage system with membrane element area of $20 \mathrm{~m}^{2}$ (enhanced oil recovery). 
Future work will focus on optimizing the feed-side operating pressure, as well as extending the methodology to handle multicomponent gas mixtures.

\section{List of symbols}

a dimensionless constant defined by Eq. (5)

A membrane area for each stage $\left(\mathrm{m}^{2}\right)$

$A_{\mathrm{t}} \quad$ total membrane area of system $\left(\mathrm{m}^{2}\right)$

$B$ permeability of the spacing materials inside the spiral-wound leaf $\left(\mathrm{m}^{2}\right)$

$b \quad$ dimensionless constant defined by Eq. (5)

$C$ dimensionless constant defined by Eq. (2)

$C^{\prime \prime} \quad$ permeate-side pressure parameter defined by Eq. (3) $\left(\mathrm{MPa}^{2} \mathrm{~s} / \mathrm{mol}\right)$

$d \quad$ thickness of membrane skin $(m)$

$d_{\mathrm{m}} \quad$ thickness of membrane leaf $(\mathrm{m})$

$f_{\text {cc }} \quad$ annual capital charge (\%/year)

$f_{\mathrm{cp}} \quad$ capital cost of gas-powered compressors (\$/ $\mathrm{KW})$

$f_{\mathrm{hv}} \quad$ sales gas gross heating value $\left(\mathrm{MJ} / \mathrm{m}^{3}\right)$

$f_{\mathrm{mh}} \quad$ capital cost of membrane housing $\left(\$ / \mathrm{m}^{2}\right.$ membrane)

$f_{\mathrm{mr}} \quad$ expense of membrane replacement $\left(\$ / \mathrm{m}^{2}\right.$ membrane)

$f_{\mathrm{mt}} \quad$ maintenance rate (\%/year)

$f_{\text {sg }} \quad$ utility and sales gas price $\left(\$ / \mathrm{Km}^{3}\right)$

$f_{\mathrm{wk}} \quad$ working capital rate $(\%)$

$F \quad$ annual process cost $\left(\$ / \mathrm{Km}^{3}\right)$

$F_{\text {cc }} \quad$ annual capital charge (\$/year)

$F_{\text {fc }} \quad$ fixed capital investment (\$)

$F_{\mathrm{mr}} \quad$ expense of membrane replacement (\$/year)

$F_{\mathrm{mt}} \quad$ maintenance expense (\$/year)

$F_{\mathrm{pl}} \quad$ value of product losses (\$/year)

$F_{\text {ut }} \quad$ cost of utilities (\$/year)

$h \quad$ dimensionless leaf-length variable

$K$ minimum number of binary variables for integer conversion

$L \quad$ membrane leaf length (m)

$M \quad$ number of quadrature points for the integral $I\left(\gamma, y_{\mathrm{r}}{ }^{\prime}\right)$

$N \quad$ permeator stage number in configuration

$N^{A} \quad$ number of membrane elements for each stage

$N^{A L} \quad$ lower bound of membrane element number

$N^{A U} \quad$ upper bound of membrane element number

$P \quad$ feed-side pressure (MPa) $p \quad$ permeate-side pressure (MPa)

$p_{\text {out }} \quad$ required outlet permeate pressure

(MPa)

$p_{0} \quad$ permeate outlet pressure for each permeator (MPa)

$Q_{1} \quad$ permeability of the more permeable component $(\mathrm{mol} / \mathrm{m} \mathrm{s} \mathrm{Pa})$

$Q_{2} \quad$ permeability of the less permeable component $(\mathrm{mol} / \mathrm{m} \mathrm{s} \mathrm{Pa})$

$R \quad$ dimensionless permeation factor defined by Eq. (8)

$R_{\mathrm{g}} \quad$ ideal gas constant $\left(\mathrm{m}^{3} \mathrm{~Pa} / \mathrm{kg} \mathrm{mol} \mathrm{K}\right)$

$S \quad$ slack variable in logic constraints

$t_{\mathrm{m}} \quad$ membrane life (years)

$t_{\mathrm{wk}} \quad$ annual working time (days/year)

$T \quad$ temperature $(K)$

$U_{\mathrm{f}} \quad$ feed gas flow rate for each permeator $(\mathrm{mol} / \mathrm{s})$

$U_{\mathrm{f} 0} \quad$ fresh feed flow rate for each permeator $(\mathrm{mol} / \mathrm{s})$

$U_{\text {f00 }}$ total fresh feed flow rate as processing capacity ( $\mathrm{mol} / \mathrm{s})$

$U_{0} \quad$ flow rate of residue gas at permeator outlet $(\mathrm{mol} / \mathrm{s})$

$U_{\mathrm{b}} \quad$ flow rate of residue gas as recycle stream $(\mathrm{mol} / \mathrm{s})$

$U_{\mathrm{p}} \quad$ flow rate of residue gas as product stream $(\mathrm{mol} / \mathrm{s})$

$U_{\mathrm{pt}} \quad$ total flow rate of residue product $(\mathrm{mol} / \mathrm{s})$

$U^{L} \quad$ upper bound on stream flow rate $(\mathrm{mol} / \mathrm{s})$

$U^{U} \quad$ lower bound on stream flow rate $(\mathrm{mol} / \mathrm{s})$

$V_{0} \quad$ permeate flow rate at permeator outlet $(\mathrm{mol} / \mathrm{s})$

$V_{\mathrm{b}} \quad$ flow rate of permeate gas as recycle stream $(\mathrm{mol} / \mathrm{s})$

$V_{\mathrm{p}} \quad$ flow rate of permeate gas as product stream $(\mathrm{mol} / \mathrm{s})$

$V_{\mathrm{pt}} \quad$ total flow rate of permeate product $(\mathrm{mol} / \mathrm{s})$

$W \quad$ membrane leaf width (m)

$W_{\text {cp }} \quad$ compressor power $(\mathrm{KW})$

$W_{\mathrm{t}} \quad$ total compressor power $(\mathrm{KW})$

$w_{j} \quad$ quadrature weights

$x \quad$ local feed-side concentration (mole fraction)

$x_{0} \quad$ bulk residue stream concentration at permeator outlet (mole fraction)

$x_{\mathrm{f}} \quad$ feed concentration for each stage (mole fraction) 
$x_{\mathrm{f} 0} \quad$ fresh feed concentration (mole fraction)

$x_{\text {out }}$ required $\mathrm{CO}_{2}$ concentration of residue product (mole fraction)

$x_{\mathrm{pt}} \quad \mathrm{CO}_{2}$ concentration of residue product (mole fraction)

$x_{\mathrm{r}} \quad$ local residue concentration along outlet end of membrane leaf (mole fraction)

$y_{0} \quad$ permeate concentration in bulk permeate stream at permeate outlet (mole fraction)

$y_{\mathrm{pt}}$

$y_{\text {out }}$

$y^{\prime}$

$y_{\mathrm{f}}^{\prime}$

$y_{j}^{\prime}$

$y_{\mathrm{r}}^{\prime}$

$\mathbf{Z}^{\mathrm{A}}$

$Z^{U b}$

$\boldsymbol{Z}^{\mathrm{Uf0}}$

$Z^{U \mathrm{p}}$

$Z^{V \mathrm{~b}}$

$Z^{V \mathrm{p}}$

$\alpha$

$\gamma$

$\gamma_{0}$

$\mu$

$\theta_{0}$

$\Theta$

$\xi_{j}$

$\phi$

$\phi_{\mathrm{r}}$

$\eta_{\mathrm{cp}}$

$\mathrm{CO}_{2}$ concentration of permeate product (mole fraction)

required $\mathrm{CO}_{2}$ concentration of permeate product (mole fraction)

local permeate concentration on the membrane surface (mole fraction)

local permeate concentration along inlet end of membrane leaf (mole fraction)

local permeate concentration at quadrature point $j$ (mole fraction)

local permeate concentration along outlet end of membrane leaf (mole fraction)

binary variable used to express discrete membrane area

binary variable denoting the existence or nonexistence of $U_{\mathrm{b}}$

binary variable denoting the existence or nonexistence of $U_{\mathrm{f} 0}$

binary variable denoting the existence or nonexistence of $U_{\mathrm{p}}$

binary variable denoting the existence or nonexistence of $V_{\mathrm{b}}$

binary variable denoting the existence or nonexistence of $V_{\mathrm{p}}$

$=Q_{1} / Q_{2}$, membrane selectivity

$=p / P$, ratio of permeate pressure to feed pressure

$=p_{0} / P$, ratio of permeate pressure to feed pressure at permeate outlet

viscosity of gas mixture ( $\mathrm{Pa} \mathrm{s}$ )

$V_{0} \quad V_{0} / U_{\mathrm{f}}$, ratio of permeate flow to feed flow at permeate outlet

\section{$\mathrm{CH}_{4}$ recovery (\%)}

quadrature points

dimensionless feed-side flow rate

dimensionless feed-side flow rate at residue outlet

compressor efficiency (\%)

\subsection{Subscripts}

$i \quad$ index of quadrature points for leaf length variable $h$

$j \quad$ index of quadrature points for integral function Eq. (9)

$k \quad$ index of binary variables in expression of discrete membrane area

$m \quad$ index of membrane stages

$n \quad$ index of membrane stages

\section{Acknowledgements}

Financial support from the LSU Office of Research and Development and Praxair, as well as technical support from the GAMS Development Corporation, are gratefully acknowledged.

\section{Appendix A}

\section{A.1. Permeator Model Constraints}

In the permeator model, the following equations are used to define parameters:

$$
\begin{aligned}
& C_{n}=C^{\prime \prime} U_{\mathrm{f}, n} /\left(A_{n} P^{2}\right), \quad n=1, \ldots, N \\
& R_{n}=\left(Q_{2} / d\right) A_{n} P / U_{\mathrm{f}, n}, \quad n=1, \ldots, N \\
& V_{0, n}=U_{\mathrm{f}, n} \theta_{0, n}, \quad n=1, \ldots, N \\
& p_{0, n}=P \gamma_{0, n}, \quad n=1, \ldots, N \\
& a_{n}=\frac{\gamma_{n}(\alpha-1)+1}{(\alpha-1)\left(1-\gamma_{n}\right)}, \quad n=1, \ldots, N \\
& b_{n}=\frac{\gamma_{n}(\alpha-1)-\alpha}{(\alpha-1)\left(1-\gamma_{n}\right)}, \quad n=1, \ldots, N
\end{aligned}
$$

The permeate-side pressure distribution is obtained from Eq. (1) using a single quadrature point at $h_{1}=0.5$

$$
\gamma_{n}^{2}=\gamma_{0, n}^{2}+0.375 C_{n}\left(1-\phi_{\mathrm{r}}, n\right), \quad n=1, \ldots, N
$$

The dimensionless feed-side flow rate $\phi$ at each quadrature point $j$ and the residue outlet, and the integral term $I$ are expressed as follows:

$$
\begin{gathered}
y_{j, n}^{\prime}=y_{\mathrm{f}, n}^{\prime}+\xi_{j}\left(y_{\mathrm{r}, n}^{\prime}-y_{\mathrm{f}, n}^{\prime}\right), \quad j=1, \ldots, M \\
n=1, \ldots, N
\end{gathered}
$$


$\phi_{j, n}=\left(\frac{y_{j, n}^{\prime}}{y_{\mathrm{f}, n}^{\prime}}\right)^{a_{n}}\left(\frac{1-y_{j, n}^{\prime}}{1-y_{\mathrm{f}, n}^{\prime}}\right)^{b_{n}}\left(\frac{\alpha-(\alpha-1) y_{j, n}^{\prime}}{\alpha-(\alpha-1) y_{\mathrm{f}, n}^{\prime}}\right)$,

$$
j=1, \ldots, M ; \quad n=1, \ldots, N
$$

$\phi_{\mathrm{r}, n}=\left(\frac{y_{\mathrm{r}, n}^{\prime}}{y_{\mathrm{f}, n}^{\prime}}\right)^{a_{n}}\left(\frac{1-y_{\mathrm{r}, n}^{\prime}}{1-y_{\mathrm{f}, n}^{\prime}}\right)^{b_{n}}\left(\frac{\alpha-(\alpha-1) y_{\mathrm{r}, n}^{\prime}}{\alpha-(\alpha-1) y_{\mathrm{f}, n}^{\prime}}\right)$,

$$
n=1, \ldots, N
$$

$I_{n}=\left(y_{\mathrm{r}, n}^{\prime}-y_{\mathrm{f}, n}^{\prime}\right) \sum_{j=1}^{M} \phi_{j, n} w_{j}, \quad n=1, \ldots, N$

The relation Eq. (8) for the dimensionless permeation factor is written as:

$$
\begin{aligned}
\alpha\left(1-\gamma_{n}\right) R_{n}= & \alpha-(\alpha-1) y_{\mathrm{f}, n}^{\prime} \\
& -\left[\alpha-(\alpha-1) y_{\mathrm{r}, n}^{\prime}\right] \phi_{\mathrm{r}, n}-(\alpha-1) I_{n}, \\
& n=1, \ldots, N
\end{aligned}
$$

The relation Eq. (11) is needed for both feed and residue ends of the permeator:

$$
\begin{aligned}
& \frac{y_{\mathrm{f}, n}^{\prime}}{1-y_{\mathrm{f}, n}^{\prime}}=\frac{\alpha\left(x_{\mathrm{f}, n}-\gamma_{n} y_{\mathrm{f}, n}^{\prime}\right)}{1-x_{\mathrm{f}, n}-\gamma_{n}\left(1-y_{\mathrm{f}, n}^{\prime}\right)}, \quad n=1, \ldots, N \\
& \frac{y_{\mathrm{r}, n}^{\prime}}{1-y_{\mathrm{r}, n}^{\prime}}=\frac{\alpha\left(x_{\mathrm{r}, n}-\gamma_{n} y_{\mathrm{r}, n}^{\prime}\right)}{1-x_{\mathrm{r}, n}-\gamma_{n}\left(1-y_{\mathrm{r}, n}^{\prime}\right)}, n=1, \ldots, N
\end{aligned}
$$

The flow rate and concentration of the effluent permeate stream from each stage are given by Eqs. (12) and (13):

$$
\begin{aligned}
& \theta_{0, n}=1-\phi_{\mathrm{r}, n}, \quad n=1, \ldots, N \\
& y_{0, n}=\frac{x_{\mathrm{f}, n}-x_{\mathrm{r}, n} \phi_{\mathrm{r}, n}}{1-\phi_{\mathrm{r}, n}}, \quad n=1, \ldots, N
\end{aligned}
$$

The flow rate and concentration of the effluent residue stream are constrained by material balance equations.

\section{A.2. Logic constraints}

Logic constraints are placed on binary variables associated with the existence or non-existence of various interconnections. The first type of logic constraint forces the flow rate to be zero if the associated connection is not utilized $(\boldsymbol{Z}=0)$. If the connection is utilized $(\boldsymbol{Z}=1)$, the corresponding constraint is relaxed to allow the flow rate to assume any value up to an upper bound $\left(U^{U}\right)$. These logic relations for the feed, product, and recycle streams are expressed as follows:

$$
\begin{aligned}
& U_{\mathrm{f} 0, n}-U^{U} \boldsymbol{Z}_{n}^{U \mathrm{f0}} \leq 0, \quad n=1, \ldots, N \\
& U_{\mathrm{p}, n}-U^{U} \boldsymbol{Z}_{n}^{U \mathrm{p}} \leq 0, \quad n=1, \ldots, N \\
& V_{\mathrm{p}, n}-U^{U} \boldsymbol{Z}_{n}^{V \mathrm{p}} \leq 0, \quad n=1, \ldots, N \\
& U_{\mathrm{b}, m, n}-U^{U} \boldsymbol{Z}_{m, n}^{U \mathrm{~b}} \leq 0, \quad m=1, \ldots, N ; \quad n=1, \ldots, N
\end{aligned}
$$

$V_{\mathrm{b}, m, n}-U^{U} \boldsymbol{Z}_{m, n}^{V \mathrm{~b}} \leq 0, \quad m=1, \ldots, N ; \quad n=1, \ldots, N$

The second type of logic constraint forces the binary variable to be zero if the associated flow rate becomes zero. If the connection is utilized, the corresponding flow rate can assume any value greater than a lower bound $\left(U^{L}\right)$. These logic relations are expressed as:

$U_{\mathrm{f} 0, n}-U^{L} Z_{n}^{U \mathrm{f} 0} \geq 0, \quad n=1, \ldots, N$
$U_{\mathrm{p}, n}-U^{L} Z_{n}^{U \mathrm{p}} \geq 0, \quad n=1, \ldots, N$
$V_{\mathrm{p}, n}-U^{L} \boldsymbol{Z}_{n}^{V \mathrm{p}} \geq 0, \quad n=1, \ldots, N$
$U_{\mathrm{b}, m, n}-U^{L} \boldsymbol{Z}_{m, n}^{U \mathrm{~b}} \geq 0, \quad m=1, \ldots, N ; \quad n=1, \ldots, N$

$V_{\mathrm{b}, m, n}-U^{L} \boldsymbol{Z}_{m, n}^{V \mathrm{~b}} \geq 0, \quad m=1, \ldots, N ; \quad n=1, \ldots, N$

In practice, $U^{L}$ is a small positive value which is chosen as the minimum flow rate allowed in the system.

The final type of logic constraint is associated with the outlet permeate pressure for each stage. If the outlet permeate stream goes to the final product stream mixer, the permeate pressure must equal the product pressure. If the permeate stream is recycled to another stage, the permeate pressure can assume any value less than or equal to the feed-side pressure. By introducing a slack variable $S$, these logic relations can expressed as:

$p_{0, n}=S_{n}+p_{\text {out }}, \quad n=1, \ldots, N$
$0 \leq S_{n} \leq\left(P-p_{\text {out }}\right)\left(1-Z_{n}^{V \mathrm{p}}\right), \quad n=1, \ldots, N$

Note that if $\boldsymbol{Z}_{n}^{V \mathrm{p}}=1$, then $S_{n}=0$ and $p_{0, n}=p_{\text {out }}$; if $Z_{n}^{V \mathrm{p}}=0$, then $0 \leq S_{n} \leq P-p_{\text {out }}$ and $p_{\text {out }} \leq p_{0, n} \leq P$. 


\section{A.3. Non-negativity and integrality constraints}

These constraints are used to specify the lower and upper variable bounds to prevent undefined operations (for example, division by zero) and to ensure that the variables remain in a reasonable solution space. Proper selection of these bounds is very important for the efficient solution of mixed-integer nonlinear models. These constraints are expressed as follows:

$$
\begin{aligned}
& 0 \leq U_{\mathrm{f} 0, n}, \quad U_{\mathrm{f}, n}, \quad U_{0, n}, \quad V_{0, n}, \quad U_{\mathrm{p}, n}, \quad V_{\mathrm{p}, n}, \\
& U_{\mathrm{b}, m, n}, \quad V_{\mathrm{b}, m, n}, \quad U_{\mathrm{pt}}, \quad V_{\mathrm{pt}} \leq U^{U} \\
& A_{n}, \quad N_{n}^{A}, \quad R_{n}, \quad C_{n} \geq 0 \\
& 0 \leq x_{f, n}, \quad x_{0, n}, \quad y_{0, n}, \quad y_{f, n}^{\prime}, \quad y_{r, n}^{\prime}, \quad y_{j, n}^{\prime}, \quad x_{r, n} \leq 1 \\
& 0 \leq \theta_{0, n}, \quad \gamma_{0, n}, \quad \gamma_{n}, \quad \phi_{r, n}, \quad \phi_{j, n} \leq 1 \\
& Z_{n}^{U \mathrm{ff} 0}, \quad Z_{n}^{U \mathrm{p}}, \quad Z_{n}^{V p}, \quad Z_{m, n}^{U \mathrm{~b}}, \quad Z_{m, n}^{V \mathrm{~b}}, \quad Z_{n, k}^{A}=0,1
\end{aligned}
$$

$$
\begin{aligned}
& m=1, \ldots, N ; \quad n=1, \ldots, N ; \quad j=1, \ldots, M \\
& \quad k=1, \ldots, K
\end{aligned}
$$

\section{References}

[1] R.W. Spillman, Economics of gas separation membranes, Chem. Eng. Prog. (1989) 41-62.

[2] F.J.C. Fournie, J.P. Agostini, Permeation membranes can efficiently replace conventional gas treatment processes, J. Petroleum Tech. (1987) 707-712.

[3] R.T. Chern, W.J. Koros, P.S. Fedkiw, Simulation of a hollowfiber separation: The effects of process and design variables, Ind. Eng. Chem. Des. Dev. 24 (1985) 1015-1022.

[4] R.W. Spillman, M.G. Barrett, T.E. Cooley, Gas membrane process optimization. In AIChE National Meeting, New Orleans, LA, 1988.

[5] R.E. Babcock, R.W. Spillman, C.S. Goddin, T.E. Cooley, Natural gas cleanup: A comparison of membrane and amine treatment processes, Energy Prog. 8 (1988) 135-142.

[6] B.D. Bhide, S.A. Stern, Membrane processes for the removal of acid gases from natural gas. I. Process configuration and optimization of operating conditions, J. Membrane Sci. 81 (1993) 209-237.

[7] B.D. Bhide, S.A. Stern, Membrane processes for the removal of acid gases from natural gas. II. Effect of operating conditions, economic parameters, and membrane properties, J. Membrane Sci. 81 (1993) 239-252.

[8] B.D. Bhide, S.A. Stern, A new evaluation of membrane processes for the oxygen-enrichment of air. I. Identification of optimum operating conditions and process configuration, J. Membrane Sci. 62 (1991) 13-35.

[9] B.D. Bhide, S.A. Stern, A new evaluation of membrane processes for the oxygen-enrichment of air. II. Effect of economic parameters and membrane properties, J. Membrane Sci. 81 (1991) 37-58.

[10] T. Pettersen, K.M. Lien, Design studies of membrane permeator processes for gas separation, Gas Sep. Purif. 9 (1995) 151-169.

[11] J. Xu, R. Agrawal, Gas separation membrane cascades. I. One compressor cascades with minimal energy losses due to mixing, J. Membrane Sci. 112 (1996) 115.

[12] R. Agrawal, J. Xu, Gas separation membrane cascades II. Two compressor cascades, J. Membrane Sci. 112 (1996) 129.

[13] R. Agrawal, J. Xu, Gas separation membrane cascades utilizing limited numbers of compressors, AIChE J. 42 (1996) 2141-2154.

[14] R. Agrawal, A simplified method for synthesis of gas separation membrane cascades with limited numbers of compressors, Chem. Eng. Sci. 52 (1997) 1029-1044.

[15] I.E. Grossmann, M.M. Daichendt, New trends in optimization-based approaches to process synthesis, Computers Chem. Eng. 20 (1996) 665-683.

[16] I.E. Grossmann, Z. Kravanja, Mixed-integer nonlinear programming techniques for process systems engineering, Computers Chem. Eng. 19 (1995) S189-S204.

[17] C.A. Floudas, I.E. Grossmann, Algorithmic approaches to process synthesis: Logic and global optimization, Proceedings of the Foundations of Computer-Aided Design (FOCAD'94), Snowmass, CO, 1994, p. 198-221.

[18] C.A. Floudas, Nonlinear and Mixed-Integer Optimization. Scientific Press, Oxford, UK, 1995.

[19] J. Viswanathan, I.E. Grossmann, A combined penalty function and outer-approximation method for MINLP optimization, Computers Chem. Engng. 14 (1990) 769-782.

[20] A. Brooke, D. Kendrick, A. Meeraus, GAMS: A User's Guide, Scientific Press, Palo Alto, CA, 1992.

[21] R. Qi, M.A. Henson, Approximate modeling of spiral-wound gas permeators, J. Membrane Sci. 121 (1996) 11-24.

[22] R. Qi, M.A. Henson, Modeling of spiral-wound gas permeators for multicomponent gas separations, Ind. Eng. Chem. Res. 36 (1997) 2320-2331.

[23] R. Qi, M.A. Henson, Optimization-based design of spiralwound membrane systems for $\mathrm{CO}_{2} / \mathrm{CH}_{4}$ separations, Sep. Purif. Tech. 13 (1998) 209-225.

[24] C.Y. Pan, Gas separation by permeators with high-flux asymmetric membranes, AIChE J. 29 (1983) 545-552.

[25] R.G. Rice, D.D. Do, Applied Mathematics and Modeling for Chemical Engineers, Wiley, New York, 1995.

[26] M. Zhu, M.M. El-Halwagi, M. Al-Ahmad, Optimal design and scheduling of flexible reverse osmosis networks, J. Membrane Sci. 129 (1997) 161-174.

[27] B.K. Srinivas, M.M. El-Halwagi, Optimal design of pervaporation systems for waste reduction, Computers chem. Engng. 17 (1993) 957-970.

[28] M.S. Peters, K.D. Timmerhaus, Plant Design and Economics for Chemical Engineers, McGraw-Hill, New York, 1980. 
[29] I. Quesada, I.E. Grossmann, A LP/NLP based branch and bound algorithm for convex MINLP optimization problems, Computers Chem. Engng. 16 (1992) 937-947.

[30] A.L. Lee, H.L. Feldkirchner, Development of a database for advanced processes to remove carbon dioxide from subquality natural gas. Topical Report GRI-93/0247, Gas Research Institute, 1993.
[31] A.L. Lee, H.L. Feldkirchner, S.A. Stern, A.Y. Houde, J.P. Gamez, H.S. Meyer, Field tests of membrane modules for the separation of carbon dioxide for low-quality natural gas, Gas Sep. Purif. 9 (1995) 35-43. 